\title{
SPATIAL MULTI-CRITERIA EVALUATION METHOD FOR PLANNING OF OPTIMAL ROADS ALIGNMENTS, WITH EMPHASIZE ON ROBUSTNESS ANALYSIS
}

\author{
Milan Sekulić ${ }^{1}$, Milan Marinković ${ }^{2}$, Ivan Ivković ${ }^{3}$ \\ ${ }^{1}$ Multiconsult AS Norway, NedreSkøyenvei 2, 0276 Oslo, Norway \\ ${ }^{2}$ University of Novi Sad, Department of Civil Engineering and Geodesy, Dr Sime Miloševića 12, 21102 \\ Novi Sad Serbia \\ ${ }^{3}$ University of Belgrade, Faculty of Transport and Traffic Engineering, Vojvode Stepe 305, 11000 \\ Belgrade, Serbia
}

Received 11 January 2021; accepted 18 March 2021

\begin{abstract}
The most recommended approach for road planning practice is conducting an environmental impact assessment (EIA) from the beginning of a road project, to consider economic, environmental and social impacts in the planning process. It requires a methodology by which planners and decision-makers can select a solution which has a balance among different criteria belonging to each of these groups. This can be solved by applying Spatial Multi-Criteria Evaluation (Spatial MCE) analysis. Our method consists of two parts. The first part uses a ModelBuilder, an application of ArGIS10.5, emulating Spatial MCE. The second part in our method evaluates robustness of developed model that is a main advantage of our methodology, since such evaluation has rarely been studied in Spatial MCE. Investigation of the robustness of the developed model is performed by applying One-At-a-Time (OAT) Sensitivity Analysis (SA). It has been implemented by creating stand-alone Python script that communicates with ArcGIS through the ArcPy module. The methods are applied to a case study in the Tlokweng Planning Area (TPA) in Botswana. Depending on prevailing criteria, the developed method, with slight adjustments, could be applicable to other study areas.
\end{abstract}

Keywords: ModelBuilder, ArcGIS, automated GIS-MCDA, ArcPy, road alignment.

\section{Introduction}

One of the most recommended approaches to a large road infrastructure project is conducting an Environmental Impact Assessment (EIA) during its feasibility stage (Mellberg et al., 2011; Gonzalez and Enríquez de Salamanca, 2018). EIA takes economic, environmental and social impacts into consideration before construction approval (Shah et al., 2010). In the sense of road alignment planning, conducting an EIA means identifying the most viable and optimal road alignment among different alternatives. This approach provides more benefits as opposed to other approaches in which the environmental aspect is usually considered after several road alignments have already been identified (Yakar and Celik, 2014).

${ }^{1}$ Corresponding author: milan.sekulic@multiconsult.no 
Identifying the optimal road alignment represents a spatial problem involving many usually conflicting criteria. In trying to solve this problem, the base of our proposed method is the application of spatial MultiCriteria Evaluation (Spatial MCE). Spatial MCE combines and transforms geographic (spatial) data into a final output (Malczewski, 1999). Few published papers have addressed the problems of MCE and GIS application in road corridor planning (Yakar and Celik, 2014; Rapaport and Snickars, 1999; Sadek et al., 1999; Belka, 2005; Roh et al., 2008; Effat and Hassan, 2013; Loganathan and Elangovan, 2017; Singh and Singh, 2017; Wahdan et al., 2019). Rapaport and Snickars (1999) introduced the idea of including the environmental concept at the beginning of road planning practice. Since this research, however, few papers have incorporated EIA (Belka, 2005; Effat and Hassan, 2013). Furthermore, these aforementioned publications do not include any detailed sensitivity analysis. Thus, in our approach we give special focus on the implementation of EIA in road planning practice by applying spatial MCE and sensitivity analysis. We also consider the automation of the entire process which has not been previously considered.

Our method consists of two connected parts. The first part represents the basecase scenario. In the base-case scenario, spatial MCE steps are automated using ModelBuilder application in the ArcGIS 10.5 environment. This step produces the base-case model. It incorporates identified criteria classified in three criteria groups - economic, environmental and social. Depending on criterion group preference, it produces four different solutions named as: economic, environmental, social and trade-off. Trade-off solution is based on the same preference (weight) among the three criterion groups. Manipulation of criteria and criteria groups is based on well-established mathematical structure within spatial MCE. In our approach Saaty's method of pair-wise comparisons (Analytical Hierarchy Process (AHP)) and Weighted Sum (WS) are used.

Developed model through base-case scenario does not give information about its robustness. Thus, the second part investigates robustness of the developed model in order to find which criteria display sensitivity. For this purpose, One-At-a-Time (OAT) Sensitivity Analysis is performed, in which each criterion weight obtained through the base-case scenario is changed and model output further examined. This is competed by creating stand-alone Python script. Robustness has rarely been studied in Spatial MCE, which is a main point of this paper.

\section{Structure of Developed Spatial MCE Method}

\subsection{Base-Case Development}

The main structure of the base-case scenario represents the model developed within the ModelBuilder application in the ArcGIS 10.5 environment. This model emulates the proposed spatial MCE method. The layout of the general model is shown in Fig. 1. It performs nine different steps, which are described in more detail below. These steps correspond to tasks performed by using available geoprocessing tools within ModelBuilder.

The model was further adjusted to the specific conditions for the case study for Tlokweng Planning Area (TPA) in Botswana. Current road planning practice in Botswana does not apply any spatial MCE; therefore, it was considered as a useful case study. 


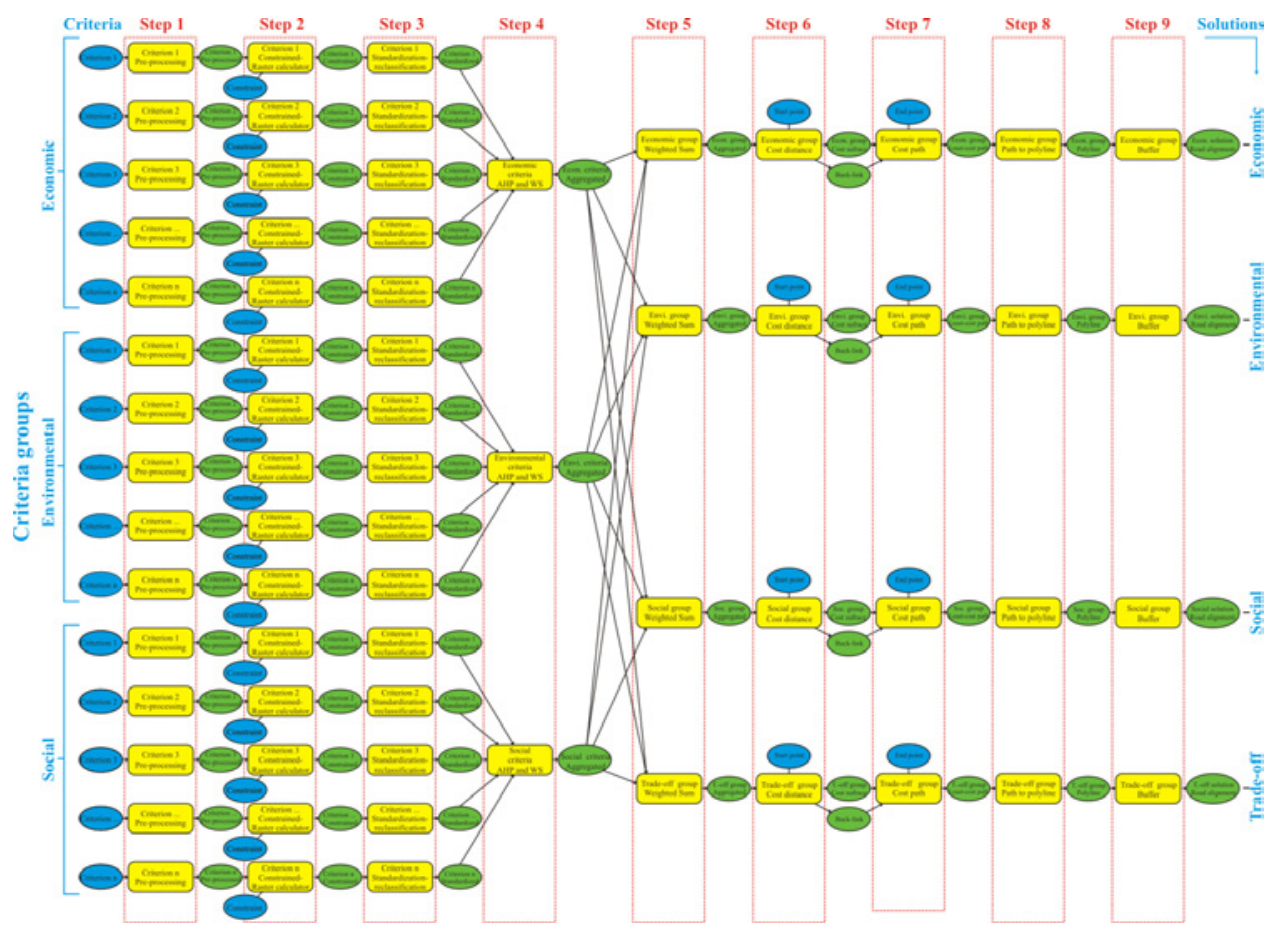

Fig. 1.

Layout of the General Model to Obtain Road Alignments by Performing Spatial MCE Method

Step 1: Pre-Processing of Input Data (Criterion Maps). The definition of the criteria is based on examination of relevant literature, expert knowledge (survey of opinions) and an analytical study (Malczewski, 1999; Malczewski and Rinner, 2015). Criteria are assigned to three criterion groups economic, environmental and social as presented in Fig. 1. Based on the prevailing condition for the case study for TPA, literature review (Yakar and Celik, 2014; Rapaport and Snickars, 1999; Sadek et al., 1999; Belka, 2005; Roh et al., 2008; Effat and Hassan, 2013; Loganathan and Elangovan, 2017; Singh and Singh, 2017; Wahdan et al., 2019) and the Botswana Road Design Manual, thirteen criteria are defined and grouped as:
- Economic criterion group (C1-Slope of the terrain, C2-Road-crossing criteria, C3-River-crossing criteria, C4-Flooding areas, C5-Terrain geology, C6-Soil type);

- Environmental criterion group (C7Natural protected areas, C8-Major river streams, C9-Surface waters, C10Ground waters); and

- Social criterion group (C11-Land cover, C12-Air pollution, C13-Noise pollution).

After criteria are identified, they are presented as criteria maps. In step 1, preprocessing of input data is necessary because different GIS data types are used in the analysis. In general, some criteria maps are 
provided as polygon datasets, thus their conversion to raster dataset is completed in this step. In this type of analysis, it is also necessary to obtain raster datasets representing certain distance from spatial objects (e.g. distance from ground waters). An important point, as a part of data preparation, refers to cell size used in input data. In respect to it, all raster datasets, used in the analysis, need to have the same cell size.

For our case study for TPA, step 1 mainly consists applying two different geoprocessing tools: Polygon to Raster and Euclidian Distance. Regarding the cell size, it was decided to use cell size of $5 \times 5$ meters. This cell size is considered to be quite detailed. The main reason for having such a small cell size lies in the fact that for the society living in TPAit was of great importance to protect existing buildings and houses. Thus, smaller size of the cells in the raster datasets was used to prevent loss of data at a coarser cell size.

Step 2: Criteria Constraint. By applying the criteria constraint in beforehand, feasible space for alternatives are determined. Thus, step 2 defines the areas where alternatives are possible. This is done using the Raster Calculator geoprocessing tool.

For our case study for TPA, the constraint criterion represents new urban expansion and economic areas that are in accordance with the spatial growth scenario developed for TPA for 2025. The constraint criterion is that road by-pass alignments cannot pass through these defined areas.

Step 3: Criteria Standardization. Criteria maps need to be transformed into comparable scales. This is known as standardization of criteria. There are different approaches to perform criteria standardization, where the most commonly used are linear scale transformation, fuzzy membership functions and expert opinion (Malczewski, 1999; Malczewski, 2000). Criteria standardization is performed using the Reclassification geoprocessing tool.

For the TPA case study, a group of ten experts discussed the criteria and decided on the standardization value for each criterion. Standardization is based on a scale of 1 to 5. Every criterion is classified into different classes and class is assigned a value from 1 to 5 . There is an inverse relationship between the value and preference, where an assigned value of 1 is seen as the most preferable choice (e.g. least cost), while 5 means the least preferable choice (e.g. highest cost).

Step 4: Definition of Criterion Weight and Aggregating Criteria. It is necessary to assign weights to criteria within each criterion group. For this purpose, many different methods may be used (e.g. ranking methods, such as: rank sum, rank reciprocal and rank exponent; rating methods such as: point allocation and ratio estimation approach, Analytical Hierarchy Process (AHP), Analytical Network Process (ANP), best worst method (BWM), full consistency method (FUCOM), etc.) (Malczewski, 1999; Greene et al., 2011; Pamučar et al., 2018; Németh et al., 2019). AHP and ANP are the most applied rating methods (Malczewski and Rinner, 2015). AHP is applied in the case study. After determining the criteria weights, it is necessary to perform their aggregation within every criterion group. To complete the criteria aggregation, Weighted Sum (WS) geoprocessing tool was applied. 
To obtain the weight coefficient for every criterion, a group of ten experts were invited to a meeting to weight the criteria based on the direct method (Linstone and Turoff, 1975). For this purpose, the Delfi approach (Linstone and Turoff, 1975) was applied; experts discussed the importance of every criterion and agreed on every criterion weight. To check the consistency of the obtained criteria weights, the consistency ratio (CR), as the part of AHP, was calculated.

Step 5: Aggregating Criteria Groups. In this step, criterion groups are aggregated. Each criterion group receives certain weight determined through AHP. Aggregation of criteria groups is based on the WS approach, and for this purpose, WS geoprocessing tool is used. The output of step 5 is a series of raster datasets representing cost surfaces with cell values in the range of 1 to 5 .

Step 6: Finding the Least Accumulative Cost Distance. This step calculates the least accumulative cost distance based on produced cost surfaces obtained through Step 5 as well as a location (source input) which represents a starting point from which the road alignment will start. The least accumulative cost distance is calculated by the help of the Dijkstra's algorithm (Dijkstra, 1959). Geoprocessing tool used in step 6 is called - Cost Distance.

The first output of step 6 represents the cost distance raster dataset providing the least accumulative cost distance from each cell to the defined source location. The second output of the Cost Distance geoprocessing tool is the direction raster dataset, called a "back-link" raster (Xu and Lathrop Jr., 1994). This raster dataset contains the information of how to get from each cell, in which the least accumulative cost distance is stored, to the source cell.

Step 7: Finding the Least-Cost Path. This step calculates the least-cost path (road alignment) from a source to a destination (end point). It uses the two output raster datasets from step 6 as well as a destination point that will be connected to the source point. Step 7 uses the Cost Path geoprocessing tool. The output of this step is a raster dataset showing the calculated road alignment. Four different alignments are produced and named as follows: economic, environmental, social and trade-off. The trade-off solution receives equal weight of $33.33 \%$ of three criterion groups.

The destination point defined in step 7 and source point defined in step 6 represents the points between which the road alignment should be placed. For the TPA case study, these two points were defined before developing the model.

Step 8: Converting Least-Cost Path to a Polyline. Step 8 converts the least-cost path raster dataset to a polyline. This step will produce four different solutions (road alignments) as polyline shapefiles.

Step 9: Defining Road Reserve Width. For different road classes, it is recommended to have a certain road reserve width. Road reserve width is also referred to as "right of way" and represents a strip of land acquired by the Road Authority for provision of road infrastructure. For the TPA case study, the road reserve width is defined as 60 meters. Thus, step 9 calculates this area around obtained polylines (road alignments) by applying the Buffer geoprocessing tool with the distance parameter of 30 meters. 


\subsection{Development of One-At-a-Time (OAT) Sensitivity Analysis (SA)}

The base-case scenario does not provide insight to the robustness of the developed model. Thus, a sensitivity analysis (SA) is applied. SA is not considered a common practice in spatial MCE studies (Malczewski, 2006; Delgado and Sendra, 2004), mainly because it is time-consuming. This represents a significant drawback as model robustness remains unknown. A main advantage of our methodology is to include SA to study the robustness of the model. SA is usually performed on criterion weight (Malczewski, 1999).
OAT SA was applied on criteria weights in the TPA case study. In this analysis only one factor, a criterion weight, is changed to identify what effects it produces on the output. The procedure was repeated for each criterion within each criterion group (Saltelli et al., 2004; Chen et al., 2010). To perform the OAT SA, a stand-alone Python script was created that communicates with ArcGIS through the ArcPy module. It partially emulates the model shown in Fig. 1 encompassing all necessary modifications to complete the SA. Fig. 2 shows the algorithm to conduct SA. Each step presented in Fig. 2 is described in more details below.

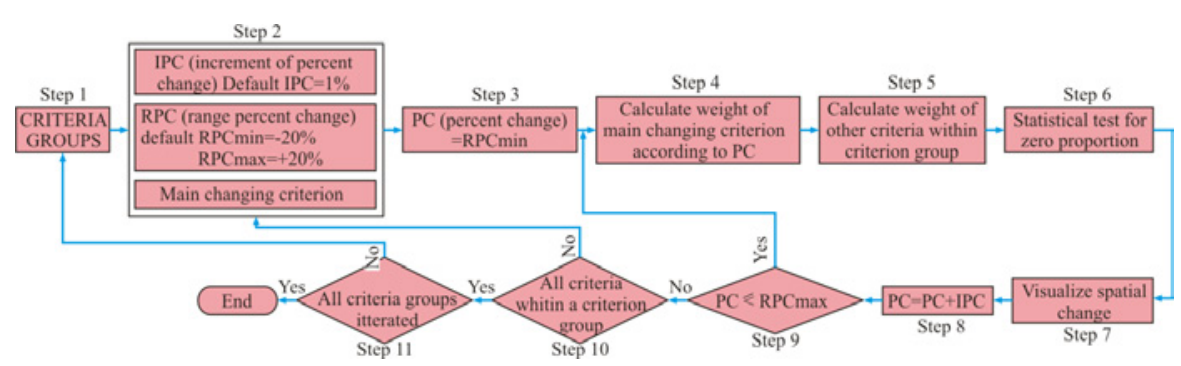

Fig. 2.

Steps to Conduct the Sensitivity Analysis

The equations presented in SA were previously applied in the MCE-focused publication of the TPA road alignment (Sekulic et al., 2020). However, the steps for SA analysis are described in detail here.

Step 1. In this step, one criterion group is first chosen among the defined three criteria groups - economic, environmental and social.

Step 2. In the second step, one criterion main changing criterion, belonging to chosen criterion group from the first step is chosen. Its initial weight is determined through
AHP in the base-case scenario. Further, in this step, the incremental percent change (IPC) and range percent change (RPC) are defined. IPC means how much percentage the criterion weight will be changed for every iteration. Default value of IPC is set up to be $1 \%$. RPC defines the range from minimum and maximum of range percent change for the criterion weight. Default value for minimum percent change - (RPCmin) is $-20 \%$ while for the maximum percent change (RPCmax) is $+20 \%$. Thus, the total number of iterations for every criterion is 41 . The total number of model runs can be calculated by Eq. (1): 
Total number of runs $=\sum_{i=1}^{n} \sum_{j=1}^{k} \sum_{m=1}^{h}(m) j(i) i$

Where $\mathrm{n}=$ the total number of criteria groups; $\mathrm{i}=$ the criterion group in question; $\mathrm{k}=$ the total number of criteria within a criterion group; $j=$ corresponding criterion in question; $h=$ the total number of incremental percentage changes for each criterion, $\mathrm{j}$; and $\mathrm{m}=$ the percent change in question.

Step 3. The first run for every criterion begins with the RPCmin. Each subsequent run increases the percent change by defined IPC in previous step 2. For our case-study in TPA, IPC takes value of $1 \%$ while RPCmin takes value of $-20 \%$.

Step 4. In this step it is necessary to recalculate the criterion weight of the main changing criterion. At any percent change, the weight of main changing criterion consists of the sum of weight defined through the AHP (base-case scenario) and percentage change in question. This is computed by Eq. (2):

$W i(j, m)=W i(j, 0)+W i(j, 0) * m$,

$m \in(R P C \min , R P C \max ),(\mathrm{i}=1 \div 3)$

Where $W i(j, m)=$ the weight for criterion, $\mathrm{j}$, for which the weight is changing within a criterion group, $\mathrm{i} ; \mathrm{Wi}(\mathrm{j}, 0)=$ the weight for base case scenario for criterion, $j$, for which the weight is changing within a criteria group, $\mathrm{i} ; \mathrm{m}=$ the percentage change within a criteria group, i; RPCmin = minimum value of range percent change - default value is $-20 \%$ and $\mathrm{RPCmax}=$ maximum value of range percent change - default value is $+20 \%$.

Step 5. In this step, the remaining criteria belonging to the same criterion group as the main changing criterion, are adjusted based on their weights defined for the base-case scenario through the AHP, by Eq. (3):

$$
\begin{aligned}
& W i(C j, m)=(1-W i(j, m)) * \frac{W i(C j, 0)}{1-W i(j, 0)}, \\
& m \in(R P C \min , R P C \max ),(\mathrm{i}=1 \div 3)
\end{aligned}
$$

Where $\mathrm{Wi}(\mathrm{Cj}, \mathrm{m})=$ the adjusted weight for criterion, $j$, within a criterion group, I, at a percentage change, $\mathrm{m} ; \mathrm{Wi}(\mathrm{j}, \mathrm{m})=$ the weight for criterion, $j$, for which the weight is changing within a criterion group, $\mathrm{i} ; \mathrm{Wi}(\mathrm{Cj}$, $0)=$ criterion weight, $\mathrm{Cj}$, for base case within a criterion group, $\mathrm{i}$; and $\mathrm{Wi}(\mathrm{j}, 0)=$ the base case criterion weight, $j$, for which the weight is changing within a criterion group, $i$.

It should be mentioned that the additivity constraint, where the sum of all criteria weights is equal to 1.0 , must be maintained as the criterion weight is adjusted. The additivity constraint at any percentage change for all criteria weights within a group is expressed by Eq. (4):

$W i(m)=\sum_{j=1}^{k} W i(j m)=1$,

$m \in(R P C \min , R P C \max ),(\mathrm{i}=1 \div 3)$

Where $\mathrm{Wi}(\mathrm{m})=$ the sum of criteria weight within a criterion group i $(i=1 \div 3)$ at percentage change $\mathrm{m} ; \mathrm{k}=$ the total number of criteria within a criterion group i; Wi(jm) $=$ the weight of criterion $j$ at a percentage change $\mathrm{m}$ within a criterion group $\mathrm{i}$; RPCmin $=$ minimum value of range percent change - default value is $-20 \%$ and RPCmax = maximum value of range percent change default value is $+20 \%$.

Step 6.After re-adjusting criteria weights in such a way that the additivity constraint is satisfied, they are further combined into criteria groups. Based on this aggregation, raster datasets are produced, representing the cost surface with cell values in the range of 1 
to 5. This is similar to step 6 described shown for the base-case scenario in Fig. 1. In the SA, for every criterion and for every iteration these cost surfaces are created. Such created raster datasets reveal two important data points. First, it gives those cells for which the cost value has not changed. Second, it gives the cells for which cost transitions have happened (for example, the cell cost value has changed from 2 to a cell cost value of 3 ). This transition among the cells is given in respect to the base-case scenario ( $0 \%$ change) for which no transition among the cells exists. In this way, the total number of changed cells is obtained in step 6 of the SA. In order to investigate the robustness of the entire model the total number of changed cells for every iteration were compared to a critical value $X_{C}$ indicating if the change in total number of cells is statistically significant. This value is based on the statistical test for zero proportion - the frequentist test (Bradley and Farnsworth, 2013). The critical value of $\mathrm{X}_{\mathrm{C}}$ is computed by Eq. (5):

$X_{c}=n p_{+}+z_{\alpha} \sqrt{n p_{+}\left(1-p_{+}\right)}$

Where $Z_{\alpha}=1.645$ for significance level $\alpha$ of $0.05, \mathrm{p}+=$ the misclassification rate taking value in the range $0.01 \div 0.03$; and $n=$ the sample size that represents the total number of cells within a cost surface raster dataset.

If the number of changed cells is higher than the computed critical value $X_{C}$, then the change in the number of cells is statistically significant. This means that the criterion weight shows sensitivity at the percent change in question.

For our case-study in TPA, the significance level $\alpha$ is chosen to be 0.05 while the misclassification rate $p+$ receives value of
0.015.The total number of cells for raster datasets with cell size of $5 \times 5$ meters is 11 343425 .

Step 7. The total number of changed cells obtained in step 6 is further visualized. This gives the spatial pattern of criterion weight sensitivity when identifying those cells within a raster dataset that have some degree of sensitivity.

Step 8. In this step, the current percent change (PC) is increased by an IPC.

Step 9. Step 9 checks if the current PC is less than or equal to RPCmax (default value of $+20 \%$ ). If this condition is not satisfied it performs a new run, in which the criterion weight in question is increased by an IPC. Otherwise, it goes to step 10. The default value for RPCmax of $+20 \%$ is also used our case-study in TPA.

Step 10. Step 10 checks if the runs for all criteria within a corresponding criterion group are completed. If not, then it goes back to step (2) and takes another criterion within the same criterion group. Otherwise, it goes to step (11).

Step 11. Step 11 performs the check over the criteria group. If this check is not satisfied, then it goes back to step (1) and takes another criterion group. Otherwise, all the runs are completed. In general, three loops are implemented in the algorithm to conduct the SA. In the first loop, 41 iterations were performed for a criterion in question. The second loop iterated over every criterion within a corresponding criterion group. Finally, the third loop iterated over every criterion group - economic, environmental and social. 


\section{Case Study}

The spatial MCE and OAT SA models were applied for the TPA case study. According to the "Tlokweng Development Plan 2025 ", two by-pass roads are considered. The main goal is to divert heavy vehicles from travelling through the built-up area of
TPA and the city center of Gaborone. The proposed by-pass road alignments were planned without any application of spatial MCE (shown in Fig. 3). Thus, the spatial MCE approach we developed was used to compare the GIS-produced solutions and the proposed by-pass alignments (Sekulic et al., 2020).

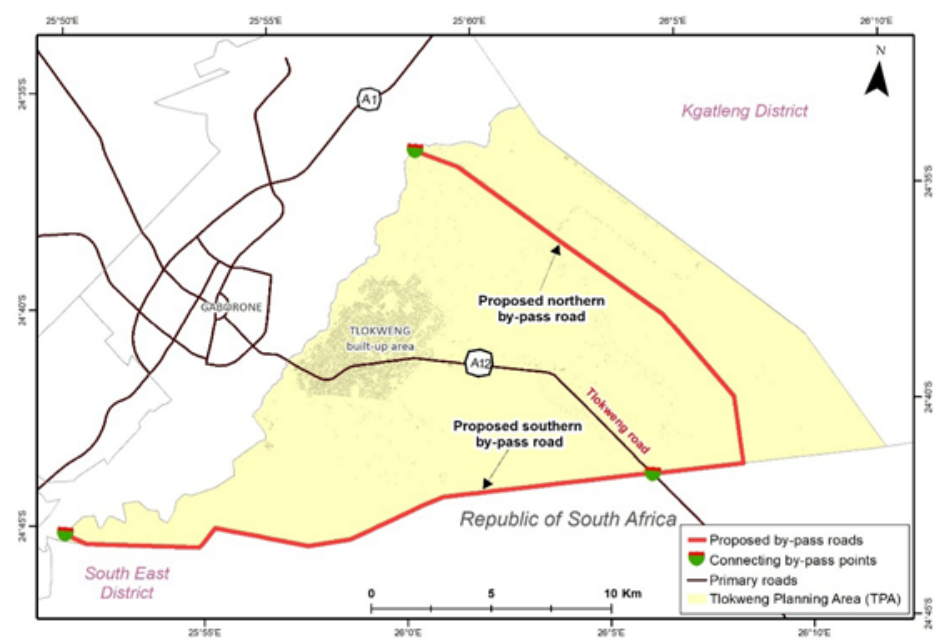

Fig. 3.

Tlokweng Planning Area (TPA) and Planned By-Pass Roads

\section{Results and Discusion}

\subsection{Output Results for the Base-Case Scenario}

Running the spatial MCE model (Fig. 1) produces four different solutions for road alignments. If the economic criterion group is preferable, and hence receives the highest weight when aggregating three criteria groups, then the final solution is called economic. The same applies to environmental and social solution. The fourth, trade-off solution, represents the road alignment for which the three criteria groups receive equal weights when aggregating them.

Fig. 4 shows the economic solution obtained after running the spatial MCE model. Two by-pass road alignments (c-routes; shown in Fig. 4) are located in the southern and northern regions of the TPA. Depending on available spatial data, one can use the solutions produced by the model to make comparison and ranking of alternatives. The detailed analysis is given in separate paper (Sekulic et al., 2020). 
The alternatives produced by the spatial MCE are suitable to be considered during the feasibility stage of the road planning process. After ranking the solutions, the most viable is chosen and this one is used for more detailed road planning. This includes geometric design such as: vertical and horizontal curves, road profile; traffic and transportation studies; detailed material investigations, etc.

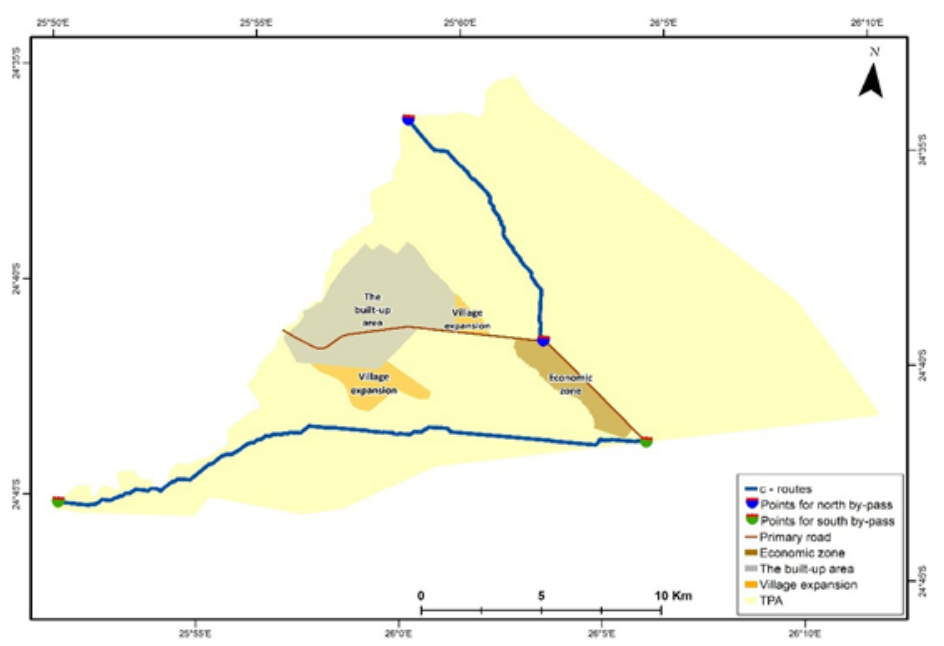

Fig. 4.

By-Pass Alignments for Economic Solution (C-Routes)

\subsection{Output Results for OAT Sensitivity Analysis}

SA performs model runs 41 times for every criterion. Thus, for the TPA case study, the total number of SA model iterations was 533. For every criterion and for every iteration, the main results produced by $S A$ are as follows:

- The number of cells for which the cost value (from 1 to 5) has not been changed and the cells for which cost transitions have happened (for example, the cell cost value has changed from 2 to a cell cost value of 3 ). Based on changed cells, total number of changed cells is also computed. Table A1 in the Appendix 1 gives the example of such table for criterion C1-slope. In Table A1, the total number of changed cells is given in column (TC);

- Raster dataset that visualizes where the cells changes have happened revealing spatial pattern of weight sensitivity.

As mentioned before, it is possible to choose different cell size for raster datasets for both base-case scenario as well as for SA. For TPA, the cell size was chosen to be $5 \times 5$ meters. One can also be interested to compare the output results with coarser resolution of raster datasets. In this paper, we also used 
the cell size of $60 \times 60$ meters, and compare SA output results for the two cases.

Table 1 summarizes the OAT SA results applied to the $5 \times 5$-meter model. The total number of cells within the $5 \times 5$-meter raster dataset covering the study area is 11343 425. The critical value (column Xc; Table 1 ) is based on the statistical test for zero proportion (Eq. (5)) and is calculated to be 170835 . Further, the total number of changed cells is presented for every criterion (columns C1,....,C13, 13 criteria in total) for every iteration (column PC - percent change). The base-case scenario represents the iteration when the $\mathrm{PC}$ receives value of $0 \%$. The results reveal four criteria as sensitive: C1 - slope, C7 - natural protected areas, C 9 - surface water and C11 - land cover. In Table 1, the iterations when criteria show sensitivity, are marked with grey color. It can be seen that criterion $\mathrm{C} 11$ - land cover is the first criterion that starts to show sensitivity at criterion weight $-5 \%$. The highest sensitivity of $\mathrm{C} 11$ is at $+8 \%$ with over two million cell transitions. The least sensitive is criterion C9 - surface water, which shows sensitivity when its criterion weight is changed by $-19 \%$ in comparison to the base-case scenario.

Table 2 summarizes the OAT SA results applied to the $60 \times 60$-meter model where the total number of cells is 78745 . The calculated critical value, $\mathrm{Xc}$, is 1237 . In
Table 2, the iterations when criteria show sensitivity, are also marked with grey color. Two differences are apparent with the larger cell size. First, when the cell size is larger, the number of sensitive criteria decreases. Sensitive criteria are: $\mathrm{C} 1$ - slope, $\mathrm{C} 7$ - natural protected areas and C11 - land cover. Criterion C9 - surface water does not show sensitivity due to aggregated or loss of data with lower resolution data. Second, the criterion weight must change by a greater value (percent change) to exhibit sensitivity. Criterion C1 - slope shows sensitivity when its criterion weight is changed by $-16 \%$ in the $60 \times 60$-meter model as opposed to $-15 \%$ in the $5 \times 5$-meter model.

Every iteration in the SA results in a raster dataset of the cost surface, which shows the spatial pattern of criterion weight sensitivity. Cells with some degree of sensitivity may be identified. Fig. 5 compares SA results of the $5 \times 5$-meter and $60 \times 60$-meter models of the $\mathrm{C} 1$ - slope criterion. Fig. 5 (a-b) compares the base-case scenario between cell lengths of 5 and 60 meters, respectively. Fig. 5 (c-d) compares the scenario with highest changed cells between cell lengths of 5 and 60 meters, respectively. The locations where the total number of cell changes is highest can be identified. C1, slope of the terrain, is most responsive to cell changes from a cost value of 4 to a cost value of 3 (Fig. 5 (c-d)). This area is located in the southwestern part of the study area within hilly terrain. 

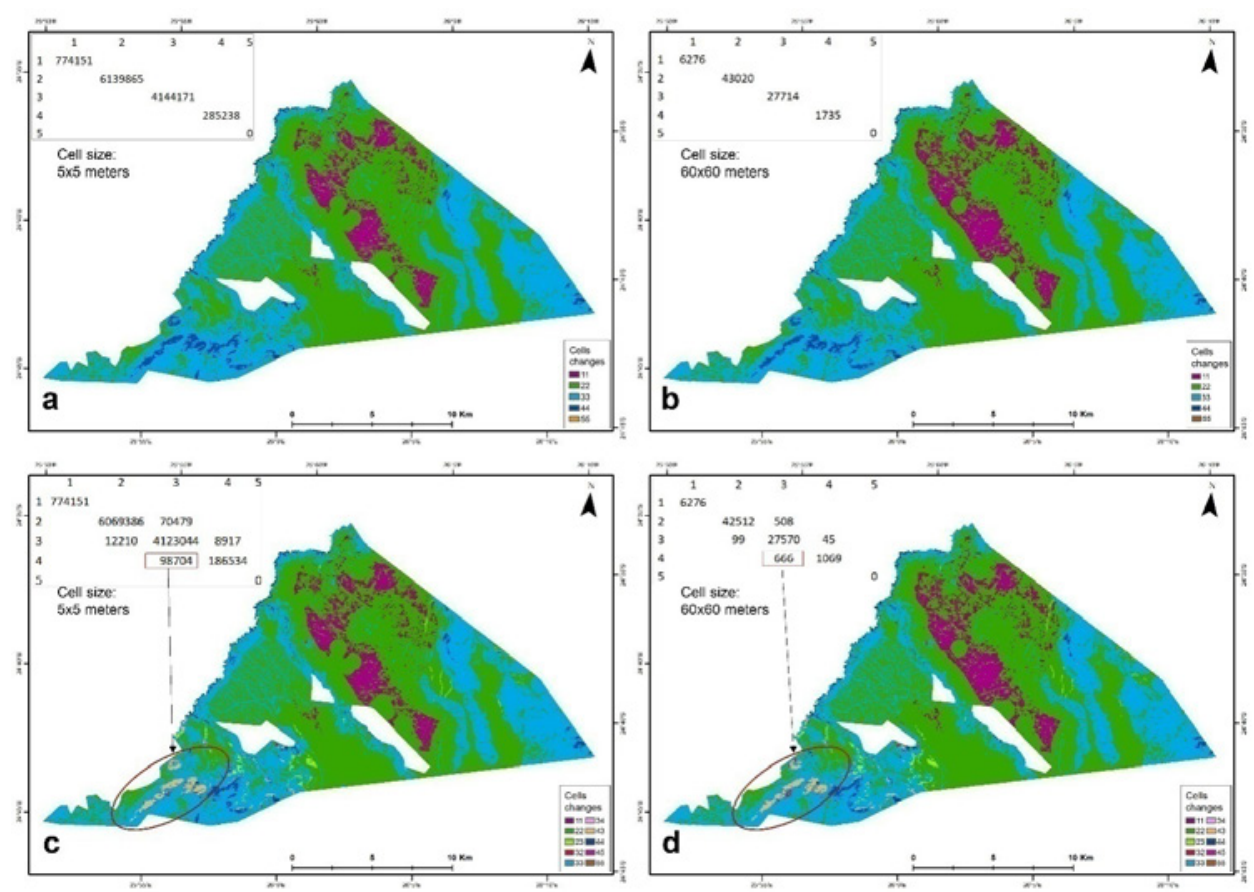

Fig. 5.

Comparison of Results for Sensitivity Analysis: Cell Size of 5 Meters (A and C) and Cell Size of 60 Meters (B and D) For Criterion C1-Slope 


\section{Table 1}

Entire Model Robustness Results with Cell Size of 5 X 5 Meters

\begin{tabular}{|c|c|c|c|c|c|c|c|c|c|c|c|c|c|c|}
\hline PC & $\mathbf{X c}$ & C1 & $\mathrm{C2}$ & $\mathrm{C} 3$ & $\mathrm{C} 4$ & $\mathrm{C5}$ & C6 & C7 & $\mathrm{C8}$ & C9 & C10 & C11 & C12 & $\mathrm{C} 13$ \\
\hline-20 & 170824 & 190310 & 29357 & 54548 & 24382 & 57177 & 46510 & 61487 & 93127 & 181900 & 129204 & 608141 & 41305 & 39691 \\
\hline-19 & 170824 & 190197 & 29357 & 54478 & 24375 & 56999 & 46507 & 61487 & 93127 & 181298 & 129204 & 608141 & 41305 & 39691 \\
\hline-18 & 170824 & 180859 & 29357 & 54478 & 24375 & 56999 & 46507 & 29902 & 93127 & 121059 & 129204 & 608141 & 41305 & 39691 \\
\hline-17 & 170824 & 180730 & 29357 & 54478 & 24321 & 55181 & 46507 & 29902 & 93127 & 119886 & 129204 & 608141 & 41305 & 39691 \\
\hline-16 & 170824 & 180730 & 27906 & 33089 & 24321 & 55181 & 8505 & 29902 & 93127 & 110238 & 127020 & 591682 & 41305 & 39691 \\
\hline-15 & 170824 & 177594 & 27906 & 33089 & 24321 & 38811 & 8505 & 29902 & 92378 & 108893 & 127020 & 591682 & 41305 & 39691 \\
\hline-14 & 170824 & 166719 & 27906 & 32367 & 24321 & 38811 & 8505 & 29902 & 92378 & 107488 & 127020 & 591682 & 41305 & 39691 \\
\hline-13 & 170824 & 166470 & 27906 & 32296 & 1073 & 38811 & 5277 & 16697 & 91260 & 107488 & 127020 & 591682 & 41305 & 39691 \\
\hline-12 & 170824 & 134201 & 27900 & 32296 & 1066 & 38811 & 5277 & 16699 & 91260 & 106063 & 125706 & 572750 & 39691 & 39691 \\
\hline-11 & 170824 & 134201 & 27900 & 32296 & 1066 & 3582 & 5277 & 15739 & 91260 & 106063 & 125706 & 572750 & 39691 & 39691 \\
\hline-10 & 170824 & 121844 & 27846 & 29782 & 1066 & 3582 & 3158 & 14890 & 91260 & 106063 & 94003 & 572750 & 39691 & 39691 \\
\hline-9 & 170824 & 121844 & 27846 & 29782 & 1066 & 3495 & 3158 & 14892 & 91260 & 103640 & 94003 & 572750 & 39691 & 39691 \\
\hline-8 & 170824 & 121844 & 350 & 29782 & 435 & 3495 & 3158 & 14890 & 90315 & 103640 & 63382 & 563530 & 39691 & 39691 \\
\hline-7 & 170824 & 2296 & 350 & 25854 & 435 & 2236 & 3155 & 12843 & 90227 & 84616 & 63382 & 563530 & 39691 & 39691 \\
\hline-6 & 170824 & 2296 & 350 & 25854 & 178 & 2236 & 3155 & 12843 & 87026 & 84616 & 63382 & 563530 & 39691 & 39691 \\
\hline-5 & 170824 & 2303 & 350 & 856 & 171 & 2236 & 693 & 12843 & 87026 & 22997 & 57464 & 563530 & 39691 & 39691 \\
\hline-4 & 170824 & 1951 & 350 & 856 & 85 & 2236 & 693 & 4260 & 56405 & 22997 & 56405 & 3321 & 0 & 0 \\
\hline-3 & 170824 & 1953 & 350 & 856 & 85 & 39 & 693 & 1059 & 56405 & 22441 & 56405 & 3321 & 0 & 0 \\
\hline-2 & 170824 & 70 & 264 & 450 & 85 & 39 & 273 & 1059 & 0 & 21864 & 0 & 3321 & 0 & 0 \\
\hline-1 & 170824 & 70 & 264 & 241 & 85 & 39 & 273 & 0 & 0 & 2 & 0 & 0 & 0 & 0 \\
\hline 0 & 170824 & 0 & 0 & 0 & 0 & 0 & 0 & 0 & 0 & 0 & 0 & 0 & 0 & 0 \\
\hline 1 & 170824 & 257 & 16 & 32 & 179 & 101 & 7 & 0 & 2 & 0 & 2 & 39691 & 0 & 0 \\
\hline 2 & 170824 & 257 & 16 & 32 & 172 & 101 & 7 & 0 & 2 & 57464 & 2 & 39691 & 0 & 0 \\
\hline 3 & 170824 & 1029 & 16 & 1876 & 172 & 101 & 27 & 108984 & 1542 & 66583 & 2 & 39691 & 0 & 0 \\
\hline 4 & 170824 & 4957 & 16 & 1876 & 172 & 2173 & 27 & 108984 & 1542 & 66583 & 2 & 41305 & 0 & 0 \\
\hline 5 & 170824 & 28882 & 720 & 1876 & 1824 & 2173 & 27 & 108986 & 3726 & 67969 & 2 & 41305 & 0 & 0 \\
\hline 6 & 170824 & 28882 & 720 & 3412 & 1824 & 2313 & 1494 & 128564 & 3726 & 68818 & 2 & 41305 & 3321 & 0 \\
\hline 7 & 170824 & 28882 & 720 & 3412 & 2528 & 2173 & 1494 & 128564 & 3726 & 68906 & 2 & 41305 & 3321 & 0 \\
\hline 8 & 170824 & 29320 & 720 & 4293 & 2535 & 25483 & 22856 & 128566 & 3726 & 68906 & 2 & 2169445 & 3321 & 0 \\
\hline 9 & 170824 & 29320 & 1522 & 4293 & 2870 & 25483 & 22856 & 128564 & 9644 & 70316 & 21864 & 2169445 & 3321 & 0 \\
\hline 10 & 170824 & 33896 & 1522 & 4293 & 2870 & 55669 & 22856 & 221886 & 9644 & 87418 & 21864 & 2169445 & 3321 & 0 \\
\hline 11 & 170824 & 33896 & 1522 & 18630 & 2870 & 57307 & 58866 & 221886 & 9644 & 87418 & 21864 & 2169445 & 3321 & 0 \\
\hline 12 & 170824 & 37355 & 5451 & 18630 & 2870 & 57307 & 58866 & 221886 & 9644 & 115366 & 21864 & 2169445 & 3321 & 0 \\
\hline 13 & 170824 & 37355 & 5451 & 18808 & 2870 & 65867 & 58866 & 221886 & 10958 & 115366 & 21864 & 2169445 & 3321 & 0 \\
\hline 14 & 170824 & 40745 & 5451 & 24647 & 27634 & 65867 & 58866 & 221886 & 10958 & 116804 & 21864 & 2169445 & 3321 & 0 \\
\hline 15 & 170824 & 41088 & 5451 & 24647 & 27641 & 68825 & 58866 & 222227 & 10958 & 116872 & 21864 & 2169445 & 3321 & 0 \\
\hline 16 & 170824 & 41088 & 7436 & 153209 & 27634 & 68825 & 59335 & 222227 & 10958 & 122995 & 23194 & 2169445 & 3321 & 0 \\
\hline 17 & 170824 & 41727 & 7436 & 153209 & 27634 & 68825 & 59335 & 222227 & 72577 & 122995 & 23194 & 2169445 & 3321 & 0 \\
\hline 18 & 170824 & 58854 & 7436 & 153211 & 27634 & 73739 & 59471 & 222227 & 73199 & 131053 & 23194 & 2169445 & 3321 & 0 \\
\hline 19 & 170824 & 58854 & 7436 & 153218 & 27634 & 73739 & 59471 & 222228 & 73199 & 131069 & 23194 & 2169445 & 3321 & 0 \\
\hline 20 & 170824 & 58861 & 7614 & 153211 & 27634 & 73739 & 59471 & 222228 & 73199 & 144274 & 23194 & 2169445 & 3321 & 0 \\
\hline
\end{tabular}




\section{Table 2}

Entire Model Robustness Results with Cell Size of 60 X 60 Meters

\begin{tabular}{|c|c|c|c|c|c|c|c|c|c|c|c|c|c|c|}
\hline PC & Xc & 1 & $\mathrm{C2}$ & $\mathrm{C} 3$ & $\mathrm{C} 4$ & $\mathrm{C5}$ & C6 & C7 & $\mathrm{C} 8$ & C9 & $\mathrm{C} 10$ & C11 & C12 & C13 \\
\hline-20 & 237 & 318 & 173 & 349 & 139 & 400 & 325 & 274 & 802 & 834 & 988 & 3856 & 189 & 189 \\
\hline-19 & 237 & 316 & 173 & 347 & 130 & 399 & 325 & 274 & 802 & 833 & 988 & 3856 & 189 & 189 \\
\hline-18 & 237 & 253 & 173 & 347 & & & & 139 & 802 & 635 & 988 & 3856 & 189 & 189 \\
\hline-17 & 237 & & 173 & 347 & & 385 & 325 & 139 & 802 & 626 & 988 & 856 & 189 & 89 \\
\hline-16 & 237 & 252 & 162 & 200 & 139 & 385 & 66 & 139 & 802 & 546 & 979 & 3738 & 189 & 189 \\
\hline-15 & 237 & 229 & 162 & 200 & 139 & 265 & 66 & 139 & 797 & 527 & 979 & 3738 & 189 & 189 \\
\hline-14 & 1237 & 144 & 162 & 196 & 139 & 265 & 66 & 139 & 797 & 518 & 979 & 3738 & 189 & 189 \\
\hline-13 & 1237 & 44 & 162 & 195 & 8 & 265 & 42 & 86 & 786 & 518 & 979 & 738 & 189 & 189 \\
\hline-12 & 1237 & & 162 & 195 & 8 & 265 & 42 & & 786 & 501 & 74 & 721 & 39 & 89 \\
\hline-11 & 237 & 910 & 162 & 195 & 8 & 30 & 42 & 81 & 786 & 501 & 974 & 3721 & 189 & 189 \\
\hline-10 & 1237 & 020 & 162 & 178 & 0 & 30 & 23 & & 786 & 501 & 808 & 3721 & 189 & 189 \\
\hline-9 & 1237 & 820 & 162 & 178 & 8 & 30 & 23 & & 786 & 479 & 808 & 3721 & 189 & 189 \\
\hline-8 & 1237 & 828 & 7 & 178 & 5 & 30 & 23 & 74 & 779 & 479 & 695 & 3662 & 189 & 189 \\
\hline-7 & 1237 & 15 & 7 & 148 & 5 & 21 & 23 & & 779 & & 95 & & & 189 \\
\hline-6 & 1237 & 15 & 7 & 148 & 7 & 21 & 23 & & 770 & 420 & & 3662 & 189 & 189 \\
\hline-5 & 1237 & 15 & 7 & 9 & 4 & 21 & 9 & 65 & 770 & 198 & 657 & 3662 & 189 & 189 \\
\hline-4 & 1237 & 1 & 7 & 9 & 3 & 21 & 9 & & 657 & 198 & 657 & 41 & 0 & 0 \\
\hline-3 & 1237 & 11 & 7 & 9 & 3 & 1 & 9 & 0 & 657 & 194 & 657 & 41 & 0 & 0 \\
\hline-2 & 1237 & 0 & 6 & 6 & 5 & 1 & 6 & & 0 & 185 & 0 & 41 & 0 & 0 \\
\hline-1 & 1237 & 0 & 6 & 5 & 3 & 1 & 6 & & 0 & 3 & 0 & 0 & 0 & 0 \\
\hline 0 & 1237 & 0 & 0 & 0 & ( & 0 & c & & 0 & c & ( & & & 0 \\
\hline 1 & 1237 & 6 & 0 & 1 & 3 & 3 & 0 & 0 & 3 & & & 189 & & 0 \\
\hline 2 & 1237 & 6 & 0 & 1 & 3 & 3 & 0 & c & 3 & & 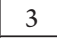 & 189 & 0 & 0 \\
\hline 3 & 1237 & 11 & 0 & 12 & 3 & 3 & 0 & 9 & 12 & 70 & 3 & 189 & 0 & 0 \\
\hline 4 & 1237 & 41 & 0 & 12 & & 1 & 0 & 52 & 12 & 704 & 3 & 189 & & 0 \\
\hline 5 & 1237 & & 6 & 12 & & 12 & 0 & 055 & 21 & 712 & & 180 & 0 & 0 \\
\hline 6 & 1237 & & 6 & & & 14 & & & & 719 & & & & 0 \\
\hline 7 & 1237 & & 6 & 23 & $1 c$ & 12 & 1 & & 21 & 71 & 3 & & 41 & 0 \\
\hline 8 & 1237 & 182 & 6 & 28 & 16 & 142 & 157 & 1018 & 21 & 719 & 3 & 14436 & 41 & 0 \\
\hline 9 & 1237 & 182 & 11 & 28 & & 142 & 157 & 1015 & 59 & 725 & 185 & & 41 & 0 \\
\hline 10 & 1237 & & & 28 & 20 & 365 & 157 & & 59 & 790 & & & 41 & 0 \\
\hline 11 & 1237 & & 11 & 127 & 20 & 78 & & & 59 & 790 & 185 & & 41 & 0 \\
\hline 12 & 1237 & 260 & 41 & 127 & 20 & 378 & 379 & 1403 & 59 & 1075 & 185 & 14436 & 41 & 0 \\
\hline 13 & 1237 & 260 & 41 & 128 & 20 & 437 & 379 & 1403 & 64 & 1075 & 185 & 14436 & 41 & 0 \\
\hline 14 & 1237 & 281 & 41 & 183 & 186 & 437 & 379 & 1403 & 64 & 1079 & 185 & 14436 & 41 & 0 \\
\hline 15 & 1237 & & 41 & 183 & 186 & 455 & 379 & 1407 & 64 & 1079 & 185 & 14436 & 41 & 0 \\
\hline 16 & 1237 & & 56 & 1049 & 186 & 455 & 383 & 1407 & 64 & 1098 & 201 & 14436 & 41 & 0 \\
\hline 17 & 1237 & 290 & 56 & 1049 & 186 & 455 & 383 & 1407 & 286 & 1098 & 201 & 14436 & 41 & 0 \\
\hline 18 & 1237 & 325 & 56 & 1049 & 186 & 489 & 385 & 1407 & 293 & 1132 & 201 & 14436 & 41 & 0 \\
\hline 19 & 1237 & 398 & 56 & 1049 & 186 & 489 & 385 & 1408 & 293 & 1133 & 201 & 14436 & 41 & 0 \\
\hline 20 & 1237 & 398 & 57 & 1049 & 186 & 489 & 385 & 1408 & 293 & 1186 & 201 & 14436 & 41 & 0 \\
\hline
\end{tabular}




\section{Conclusion}

Applied spatial MCE methodology as an integral part of EIA facilitates the combination of criteria in three criterion groups, economic, environmental and social, in order to find optimal road alignments. The main advantage of this approach is several different scenarios can be tested very quickly and efficiently. Different scenarios may include different criterion standardisation values, different criteria weights and different weights among the criteria groups. This approach is not strictly limited to testing a certain number of solutions. The proposed methodology is flexible in the manner in which locations of end and start points of road alignments may be modelled. The methodology is easily repeated, updated and maintained via the ModelBuilder application. By utilizing the proposed methodology, analysts and decision-makers can make reliable decisions. The main limitation of the methodology applied in ModelBuilder, however, requires the calculation of criteria weights to be completed outside of ArcGIS (e.g. (Spinlab, 2019; Bohanec, 2008; Bana e Costa et al., 2016), etc.). The presented application of the model focuses on the TPA case study; however, to apply the methodology to another area, the criteria within each criterion group may require adjustment.

The developed approach to test criteria sensitivity should be used together with basecase model. OAT SA conducted in a standalone Python script is flexible and facilitates use of different criterion standardisation values, criteria and criterion group weights. SA could be performed with different cell sizes of raster datasets (cost surface). In general, SA needs to be conducted with the same cell size used in the base-case scenario. It is worth mentioning, that selecting a smaller cell size will allow more details to be maintained in the base-case scenario and SA. However, it will take longer time to obtain results especially related to SA. Thus, this is a trade-off and it is up to an analyst to choose what is important in their studies.

The proposed spatial MCE method successfully tested the case study TPA in Botswana, in which four different solutions of by-pass road alignments are produced. Since current road planning practice in TPA does not use any spatial MCE tool, the proposed approach can help road practitioners reach many useful solutions as well as perform comparison with alternatives not produced by spatial MCE approach. This way, better and more grounded solutions could be obtained which is especially important for those countries with limited resources.

\section{References}

Bana e Costa, C.A.; De Corte, J.M.; Vansnick, J.C. 2016. On the Mathematical Foundations of MACBETH. In Multiple Criteria Decision Analysis, International Series in Operations Research \& Management Science. Springer. Germany. 233: 421-463.

Belka, M.K. 2005. Multi-Criteria Analysis and GIS Application in the Selection of Sustainable Motorway Corridor. Master Thesis. Linköpings University. Sweden. 81p.

Bohanec, M. 2008. DEXi: Program for Multi-Attribute Decision Making - User's Manual. Institut “Jozef Stefan”. Slovenia. 56p.

Bradley, J.R.; Farnsworth, D.L. 2013. Testing For a Zero Proportion, Open Journal of Statistics 3: 258-260. 
Chen, Y.; Yu, J.; Khan, S. 2010. Spatial Sensitivity Analysis of Multi-Criteria Weights in GIS-Based Land Suitability Evaluation, Environmental Modelling \& Software 25(12): 1582-1591.

Delgado, M.G.; Sendra, J.B. 2004. Sensitivity Analysis in Multicriteria Spatial Decision-Making: A Review, Human and Ecological Risk Assessment 10(6): 1173-1187.

Dijkstra, E.W. 1959. ANote on Two Problems in Connexion with Graphs, Numerische Mathematik 1(1): 269-271.

Effat, H.A.; Hassan, O.A. 2013. Designing and Evaluation of Three Alternatives Highway Routes Using the Analytical Hierarchy Process and The Least-Cost Path Analysis, Application in Sinai Peninsula, Egypt, The Egyptian Journal of Remote Sensing and Space Science 16(2): 141-151.

Gonzalez, A.; Enríquez de Salamanca, Á. 2018. Spatial Multi-Criteria Analysis in Environmental Assessment: A Review and Reflection on Benefits and Limitations, Journal of Environmental Assessment Policy and Management 20(03): 1840001-1-1840001-24.

Greene, R.; Devillers, R.; Luther, J.E.; Eddy, B.G. 2011. GIS-Based Multiple-Criteria Decision Analysis, Geography compass 5(6): 412-432.

Linstone, H.A.; Turoff, M. 1975. The Delphi Method: Techniques and Applications. Addison-Wesley Educational Publishers, Inc. USA. 620p.

Loganathan, S.; Elangovan, K. 2017. Route Alignment between the Corridors through the Applications of RS and GIS-Perundurai to Palani, Tamil Nadu, India. In Proceedings of the International Conference of Electronics, Communication and Aerospace Technology (ICECA), 2: 23-26.

Malczewski, J. 1999. GIS and Multicriteria Decision Analysis. John Wiley \& Sons, Inc. USA. 408p.

Malczewski, J. 2000. On the Use of Weighted Linear Combination Method in GIS: Common and Best Practice Approaches, Transactions in GIS 4(1): 5-22.
Malczewski, J. 2006. GIS-Based Multicriteria Decision Analysis: A Survey of the Literature, International Journal of Geographical Information Science 20(7): 703-726.

Malczewski, J.; Rinner, C. 2015. Multicriteria Decision Analysis in Geographic Information Science. Springer. Germany. 346p.

Mellberg, I.; Lingestål, I.; Andersson, M.; Stenlund, O.; Lundmark, M. 2011. Environmental Impact Assessment: Roads and Rail: Handbook Methodology. Swedish Transport Administration. Sweden. 72p.

Németh, B.; Molnár, A.; Bozóki, S.; Wijaya, K.; Inotai, A.; Campbell, J.D.; Kaló, Z. 2019. Comparison of Weighting Methods used in Multicriteria Decision Analysis Frameworks in Healthcare with Focus on Lowand Middle-Income Countries, Journal of Comparative Effectiveness Research 8(4): 195-204.

Pamučar, D.; Stević, Ž.; Sremac, S. 2018. A New Model for Determining Weight Coefficients of Criteria in MCDM Models: Full Consistency Method (FUCOM), Symmetry 10(9): 393: 22p.

Rapaport, E.; Snickars, F. 1999. GIS-Based Road Location in Sweden: A Case Study to Minimize Environmental Damage, Building Costs and Travel Time. In Geographical Information and Planning. Springer. Germany, 135-153.

Roh, T.; Lee, J.C.; Kim, H.G.; Kim, J.J.; Jung, Y.H. 2008. Construction on Decision Support System for Route Location based on GIS, The International Archives of the Photogrammetry, Remote Sensing and Spatial Information Sciences 37(B2): 555-566.

Sadek, S.; Bedran, M.; Kaysi, I. 1999. GIS Platform for Multicriteria Evaluation of Route Alignments, Journal of Transportation Engineering 125(2): 144-151.

Saltelli, A.; Tarantola, S.; Campolongo, F.; Ratto, M. 2004. Sensitivity Analysis in Practice: A Guide to Assessing Scientific Models. Wiley, Ltd. USA. 232p. 
Sekulic, M.; Siljeg, A.; Mansourian, A.; Watson, L.; Cavric, B.I. 2020. Multi-Criteria Spatial-Based Modelling for Optimal Alignment of Roadway ByPasses in the Tlokweng Planning Area, Botswana, Journal of Spatial Science. https://doi.org/10.1080/14498596.2 020.1755731 .

Shah, A.; Khan, S.; Shah, M.H.; Khan, R.; Jan, I. 2010. Environmental Impact Assessment (EIA) of Infrastructure Development Projects in Developing Countries, OIDA International Journal of Sustainable Development 1(4): 47-54.

Singh, M.P.; Singh, P. 2017. Multi-Criteria GIS Modeling for Optimum Route Alignment Planning in Outer Region of Allahabad City, India, Arabian Journal of Geosciences 10(13): 294.

Spinlab. 2019. Definite: Decision Making Software for a Finite Set of Alternatives. Available from Internet: $<$ https://spinlab.vu.nl/>.
Wahdan, A.; Effat, H.; Abdallah, N.; Elwan, K. 2019. Design an Optimum Highway Route using Remote Sensing Data and GIS-Based Least Cost Path Model, Case of Minya-Ras Ghareb and Minya-Wahat-Bawiti Highway Routes, Egypt, American Scientific Research Journal for Engineering, Technology, and Sciences (ASRJETS) 56(1): 157-181.

Xu, J.; Lathrop Jr., G.R. 1994. Improving Cost-Path in a Raster Data Format, Computer \& Geoscience 20(10): 1455-1465.

Yakar, F.; Celik, F. 2014. A Highway Alignment Determination Model Incorporating GIS and MultiCriteria Decision Making, KSCE Journal of Civil Engineering 18(6): 1847-1857.

\section{ijtte 400}




\section{Appendix 1}

\section{Table A1}

Cell Cost Changes in Raster Dataset when the Weight of Criterion C1 - Slope Changes; Cell Size is 5x5 Meters

\begin{tabular}{|c|c|c|c|c|c|c|c|c|c|c|c|c|c|c|c|c|c|c|c|c|c|c|c|c|c|c|}
\hline PC & 11 & 12 & 13 & 14 & 15 & 21 & 22 & 23 & 24 & 25 & 31 & 32 & 33 & 34 & 35 & 41 & 42 & 43 & 44 & 45 & 51 & 52 & 53 & $54 \mid 5$ & 55 & TC \\
\hline-20 & 74151 & 0 & \begin{tabular}{|l|l|}
0 \\
\end{tabular} & \begin{tabular}{|l|l|}
0 \\
\end{tabular} & 0 & 0 & 6069386 & 70479 & 0 & \begin{tabular}{l|l}
0 \\
\end{tabular} & \begin{tabular}{|l|l|}
0 \\
\end{tabular} & 12210 & 4123044 & 8917 & 0 & \begin{tabular}{|l|l|}
0 \\
\end{tabular} & \begin{tabular}{|l|l|}
0 \\
\end{tabular} & 98704 & 186534 & \begin{tabular}{l|l}
0 \\
\end{tabular} & 0 & 0 & \begin{tabular}{l|l}
0 \\
\end{tabular} & \begin{tabular}{l|l}
0 & \\
\end{tabular} & \begin{tabular}{|l|l}
0 & \\
\end{tabular} & 190310 \\
\hline 19 & 774151 & 0 & 0 & 0 & 0 & 0 & 6069386 & 70479 & 0 & 0 & 0 & 12198 & 4123056 & 8917 & 0 & 0 & 0 & 98603 & 186635 & 0 & 0 & 0 & 0 & 0 & 0 & 90197 \\
\hline 18 & 4151 & 0 & 0 & 0 & 0 & 0 & 6077952 & 1913 & 0 & 0 & 0 & 11567 & 23742 & 3862 & 0 & 0 & 0 & 98517 & 86721 & 0 & 0 & & 0 & 0 & 0 & 0859 \\
\hline 17 & 774151 & 0 & 0 & 0 & 0 & 0 & 6077952 & 61913 & 0 & 0 & 0 & 567 & 23871 & 8733 & 0 & 0 & 0 & 98517 & 6721 & 0 & 0 & 0 & 0 & 0 & 0 & 0730 \\
\hline-16 & 774151 & 0 & 0 & 0 & 0 & 0 & 6077952 & 61913 & 0 & 0 & 0 & 11567 & 4123871 & 8733 & 0 & 0 & 0 & 98517 & 186721 & 0 & 0 & 0 & 0 & 0 & 0 & 180730 \\
\hline-15 & 774151 & 0 & 0 & 0 & 0 & 0 & 6079669 & 60196 & 0 & 0 & 0 & 10176 & 4125262 & 8733 & 0 & 0 & 0 & 98489 & 186749 & 0 & 0 & 0 & 0 & 0 & 0 & 177594 \\
\hline-14 & 774151 & 0 & 0 & 0 & 0 & 0 & 6079669 & 60196 & 0 & 0 & 0 & 8695 & 4126743 & 8733 & 0 & 0 & 0 & 89095 & 196143 & 0 & 0 & 0 & 0 & \begin{tabular}{l|l}
0 & \\
\end{tabular} & \begin{tabular}{l|l} 
& \\
\end{tabular} & 166719 \\
\hline-13 & 774151 & 0 & 0 & 0 & 0 & 0 & 6079669 & 60196 & 0 & 0 & 0 & 8446 & 4126992 & 8733 & 0 & 0 & 0 & 89095 & 196143 & 0 & 0 & 0 & 0 & 0 & 0 & 166470 \\
\hline-12 & 774151 & 0 & 0 & 0 & 0 & 0 & 6110719 & 29146 & 0 & 0 & 0 & 8446 & 4128138 & 7587 & 0 & 0 & 0 & 89022 & 196216 & 0 & 0 & 0 & 0 & 0 & 0 & 134201 \\
\hline-11 & 774151 & 0 & 0 & 0 & 0 & 0 & 6110719 & 29146 & 0 & 0 & 0 & 8446 & 4128138 & 7587 & 0 & 0 & 0 & 89022 & 196216 & 0 & 0 & 0 & 0 & 0 & 0 & 134201 \\
\hline-10 & 774151 & 0 & 0 & 0 & 0 & 0 & 6122986 & 16879 & 0 & 0 & 0 & 8356 & 4128228 & 7587 & 0 & 0 & 0 & 89022 & 196216 & 0 & 0 & 0 & 0 & 0 & 0 & 121844 \\
\hline-9 & 774151 & 0 & 0 & 0 & 0 & 0 & 6122986 & 16879 & 0 & 0 & 0 & 8356 & 4128228 & 7587 & 0 & 0 & 0 & 89022 & 196216 & 0 & 0 & 0 & 0 & 0 & 0 & 121844 \\
\hline-8 & 774151 & 0 & 0 & 0 & 0 & 0 & 6122986 & 16879 & 0 & 0 & 0 & 8356 & 4128228 & 7587 & 0 & 0 & 0 & 89022 & 196216 & 0 & 0 & 0 & 0 & \begin{tabular}{l|l}
0 & \\
\end{tabular} & \begin{tabular}{l|l}
0 \\
\end{tabular} & 121844 \\
\hline-7 & 774151 & 0 & 0 & 0 & 0 & 0 & 6137763 & 2102 & 0 & 0 & 0 & 74 & 4143993 & 104 & 0 & 0 & 0 & 16 & 285222 & 0 & 0 & 0 & 0 & 0 & 0 & 2296 \\
\hline-6 & 774151 & 0 & 0 & 0 & 0 & 0 & 6137763 & 2102 & 0 & 0 & 0 & 74 & 4143993 & 104 & 0 & 0 & 0 & 16 & 285222 & 0 & 0 & 0 & 0 & 0 & 0 & 2296 \\
\hline-5 & 774151 & 0 & 0 & 0 & 0 & 0 & 6137756 & 2109 & 0 & 0 & 0 & 74 & 4143993 & 104 & 0 & 0 & 0 & 16 & 285222 & 0 & 0 & 0 & 0 & 0 & 0 & 2303 \\
\hline-4 & 774151 & 0 & 0 & 0 & 0 & 0 & 6138001 & 1864 & 0 & 0 & 0 & 72 & 4144099 & 0 & 0 & 0 & 0 & 15 & 285223 & 0 & 0 & 0 & 0 & 0 & 0 & 1951 \\
\hline-3 & 774151 & 0 & 0 & 0 & 0 & 0 & 6137994 & 1871 & 0 & 0 & 0 & 67 & 14104 & 0 & 0 & 0 & 0 & 15 & 85223 & 0 & 0 & 0 & 0 & 0 & 0 & 1953 \\
\hline-2 & 774151 & 0 & 0 & 0 & 0 & 0 & 6139865 & 0 & 0 & 0 & 0 & 67 & 4144104 & 0 & 0 & 0 & 0 & & 285235 & 0 & 0 & 0 & 0 & 0 & 0 & 70 \\
\hline-1 & 774151 & 0 & 0 & 0 & 0 & 0 & 6139865 & 0 & 0 & 0 & 0 & 67 & 4144104 & 0 & 0 & 0 & 0 & & 285235 & 0 & 0 & 0 & 0 & 0 & 0 & 70 \\
\hline 0 & 774151 & 0 & 0 & 0 & 0 & 0 & 6139865 & 0 & 0 & 0 & 0 & 0 & 4144171 & 0 & 0 & 0 & 0 & 0 & 285238 & 0 & 0 & 0 & 0 & 0 & 0 & \\
\hline 1 & 774151 & 0 & 0 & 0 & 0 & 0 & 6139668 & 197 & 0 & 0 & 0 & 0 & 4144111 & 60 & 0 & 0 & 0 & & 285238 & 0 & 0 & 0 & 0 & 0 & 0 & 257 \\
\hline 2 & 774151 & 0 & 0 & 0 & 0 & 0 & 6139668 & 197 & 0 & 0 & 0 & 0 & 4144111 & 60 & 0 & 0 & 0 & 0 & 285238 & 0 & 0 & 0 & 0 & 0 & 0 & 257 \\
\hline 3 & 774151 & 0 & 0 & 0 & 0 & 0 & 6139668 & 197 & 0 & 0 & 0 & 654 & 4143457 & 60 & 0 & 0 & 0 & 118 & 285120 & 0 & 0 & 0 & 0 & 0 & 0 & 1029 \\
\hline 4 & 774151 & 0 & 0 & 0 & 0 & 0 & 6139668 & 197 & 0 & 0 & 0 & 654 & 4143451 & 66 & 0 & 0 & 0 & 4040 & 281198 & 0 & 0 & 0 & 0 & 0 & 0 & 4957 \\
\hline 5 & 774151 & 0 & 0 & 0 & 0 & 0 & 6133807 & 6058 & 0 & 0 & 0 & 1713 & 4125387 & 17071 & 0 & 0 & 0 & 4040 & 281198 & 0 & 0 & 0 & 0 & 0 & 0 & 28882 \\
\hline 6 & 774151 & 0 & 0 & 0 & 0 & 0 & 6133807 & 6058 & 0 & 0 & 0 & 1713 & 4125387 & 17071 & 0 & 0 & 0 & 4040 & 281198 & 0 & 0 & 0 & 0 & 0 & 0 & 28882 \\
\hline 7 & 774151 & 0 & 0 & 0 & 0 & 0 & 6133807 & 6058 & 0 & 0 & 0 & 1713 & 4125387 & 17071 & 0 & 0 & 0 & 4040 & 281198 & 0 & 0 & 0 & 0 & 0 & 0 & 28882 \\
\hline 8 & 774151 & 0 & 0 & 0 & 0 & 0 & 6133742 & 6123 & 0 & 0 & 0 & 1763 & 4125336 & 17072 & 0 & 0 & 0 & 4362 & 80876 & 0 & 0 & 0 & 0 & 0 & 0 & 9320 \\
\hline 9 & 774151 & 0 & 0 & 0 & 0 & 0 & 6133742 & 6123 & 0 & 0 & 0 & 1763 & 4125336 & 17072 & 0 & 0 & 0 & 4362 & 280876 & 0 & 0 & 0 & 0 & 0 & 0 & 29320 \\
\hline 10 & 774151 & 0 & 0 & 0 & 0 & 0 & 6133742 & 6123 & 0 & 0 & 0 & 5865 & 4120895 & 17411 & 0 & 0 & 0 & 4497 & 280741 & 0 & 0 & 0 & 0 & 0 & 0 & 33896 \\
\hline 11 & 774151 & 0 & 0 & 0 & 0 & 0 & 6133742 & 6123 & 0 & 0 & 0 & 5865 & 4120895 & 17411 & 0 & 0 & 0 & 4497 & 280741 & 0 & 0 & 0 & 0 & 0 & 0 & 33896 \\
\hline 12 & 774151 & 0 & 0 & 0 & 0 & 0 & 6133683 & 6182 & 0 & 0 & 0 & 5865 & 4117495 & 20811 & 0 & 0 & 0 & 4497 & 280741 & 0 & 0 & 0 & 0 & 0 & 0 & 37355 \\
\hline 13 & 774151 & 0 & 0 & 0 & 0 & 0 & 6133683 & 6182 & 0 & 0 & 0 & 5865 & 4117495 & 20811 & 0 & 0 & 0 & 4497 & 280741 & 0 & 0 & 0 & 0 & 0 & 0 & 37355 \\
\hline 14 & 774151 & 0 & 0 & 0 & 0 & 0 & 6133683 & 6182 & 0 & 0 & 0 & 9002 & 4114336 & 20833 & 0 & 0 & 0 & 4728 & 280510 & 0 & 0 & 0 & 0 & \begin{tabular}{|l|l|}
0 \\
\end{tabular} & 0 & 40745 \\
\hline 15 & 774151 & 0 & 0 & 0 & 0 & 0 & 6133341 & 6524 & 0 & 0 & 0 & 9002 & 4114335 & 20834 & 0 & 0 & 0 & 4728 & 280510 & 0 & 0 & 0 & 0 & 0 & 0 & 41088 \\
\hline 16 & 774151 & 0 & 0 & 0 & 0 & 0 & 6133341 & 6524 & 0 & 0 & 0 & 9002 & 4114335 & 20834 & 0 & 0 & 0 & 4728 & 280510 & 0 & 0 & 0 & 0 & 0 & 0 & 41088 \\
\hline 17 & 774151 & 0 & 0 & 0 & 0 & 0 & 6133258 & 6607 & 0 & 0 & 0 & 9003 & 4113779 & 21389 & 0 & 0 & 0 & 4728 & 280510 & 0 & 0 & 0 & 0 & 0 & 0 & 41727 \\
\hline 18 & 774151 & 0 & 0 & 0 & 0 & 0 & 6126359 & 13506 & 0 & 0 & 0 & 13241 & 4106892 & 24038 & 0 & 0 & 0 & 8069 & 277169 & 0 & 0 & 0 & 0 & 0 & 0 & 58854 \\
\hline 19 & 774151 & 0 & 0 & 0 & 0 & 0 & 6126359 & 13506 & 0 & 0 & 0 & 13241 & 4106892 & 24038 & 0 & 0 & 0 & 8069 & 277169 & 0 & 0 & 0 & 0 & 0 & 0 & 58854 \\
\hline 20 & 774151 & 0 & 0 & \begin{tabular}{|l|l|}
0 \\
\end{tabular} & 0 & 0 & 6126352 & 13513 & 0 & 0 & 0 & 13241 & 4106892 & 24038 & 0 & 0 & 0 & 8069 & 277169 & 0 & 0 & 0 & 0 & 0 & 0 & 58861 \\
\hline
\end{tabular}

